\title{
La industrialización de una bebida natural a partir del tumbo andino (Passiflora mollissima) con linaza (Linum usitatissimum)
}

\author{
Isaac César Córdova Lavado* \\ Universidad de Lima. Lima, Perú
}

Recibido: 4 de julio del 2016 / Aprobado: 28 de agosto del 2016

RESUMEN: El presente artículo muestra los resultados de una investigación para la elaboración de una bebida natural a base de una fruta exótica (tumbo) con linaza, con el fin de ofrecer un producto nuevo, saludable, natural y de fácil consumo. El producto es aceptado por hombres y mujeres entre 18 a 60 años que pertenecen a los segmentos A y B de Lima Metropolitana, y será comercializado en envases de vidrio de 300 mililitros. Además, se establece que el proyecto es económica y financieramente viable.

Palabras claves: prefactibilidad / bebida natural / jugo de fruta / tumbo (fruta) / linaza

\section{Research for the industrialization of a natural drink from the Andean tumbo (Passiflora mollissima) with linseed (Linum usitatissimum)}

ABstRACT: This article presents the results of a research for the development of a natural drink made from an exotic fruit (tumbo) with linseed, in order to offer a new, healthy and natural product for easy to consume. The product is accepted in men and women aged 18 to 60 who belong to segments A and B of Lima and will be marketed in glass containers $300 \mathrm{ml}$; also it states that the project is economically and financially viable.

Keywords: prefeasibility / natural drink / fruit juice / tumbo (fruit) / flaxseed

* Correo electrónico: 20090275@aloe.ulima.edu.pe 


\section{INTRODUCCIÓN}

El trabajo de investigación abarcó el estudio de mercado, técnico, social, económico y financiero de la producción de una bebida natural a partir del tumbo andino (Passiflora mollissima) con linaza (Linum usitatissimum). En los últimos años, la tendencia de consumo en productos naturales ha experimentado un claro auge, lo cual se refleja en un crecimiento del $18 \%$ con respecto al 2013, según el Instituto Peruano de Productos Naturales (IPPN). Los hábitos de consumo también han cambiado, debido a que el consumidor está pasando por un fenómeno de migración por "la desaceleración de las ventas de la categoría gaseosas, lo que puede ser un ejemplo de esta migración hacia productos más saludables" (Gestión, 2014). Es decir, las personas se están preocupando más por su salud y calidad de vida, por lo que optan mayormente por las bebidas a base de fruta a cambio de la gaseosa. Además, hoy es común el consumo de las semillas de linaza mezcladas con yogur, emoliente, cereal, galleta, panes, entre otros alimentos.

El crecimiento en la producción de las bebidas, jugos y néctares se presenta de los años 2002 al 2013, pero en el 2009 ocurre una disminución, debido a que la economía mundial pasó por una recesión sincronizada. En el Perú, luego de tener un crecimiento del 9,8 \% en el 2008, descendió a 0,9 \% en el PBI en el año 2009 (Parodi, 2011). Sin embargo, en los siguientes años el crecimiento es sostenido (Ministerio de Producción, 2012), por causa de los hábitos de consumo cambiantes y a los efectos migratorios hacia productos saludables.

Por ello, se propuso la elaboración de una bebida a base de una fruta exótica conocida como tumbo andino (Passiflora mollissima), que es originaria de los valles interandinos y de la zona quechua ubicada en los Andes del Perú. Esta fruta será mezclada con linaza (Linum usitatissimum), cuyas propiedades son benéficas para el organismo, y endulzada naturalmente con estevia (Stevia rebaudiana Bertoni), para obtener así un producto diferenciado de las bebidas de frutas y néctares existentes en el mercado actual.

\section{METODOLOGÍA}

Se recopiló y revisó fuentes de información primaria para la investigación de mercado, cuya secuencia inicia en la investigación cualitativa con entrevistas a profundidad al mercado objetivo: se entrevistó a nueve 
consumidores ubicados en los supermercados de Wong, Metro y Plaza Vea. Se siguió con la investigación cuantitativa y el diseño de la encuesta, que se realizó a 150 personas directamente en los supermercados de Wong y Metro, lo que ayudó a la toma de decisión frente al trabajo de investigación. Además, se utilizaron fuentes secundarias como Datatrade, informes estadísticos del Ministerio de Producción, la Organización de las Naciones Unidas para la alimentación y la agricultura (FAO), Ipsos Apoyo, entre otros. Por otro lado, se tuvo el apoyo de especialistas en investigación de mercado, en calidad y tecnología de alimentos, en procesos y tecnología industrial, en evaluación de proyectos económicofinancieros, entre otros.

\section{ESTUDIO DE MERCADO}

El producto propuesto para el proyecto es una bebida natural a base de la Passiflora mollissima H. B. K., comercialmente conocida como tumbo, mezclada con linaza (Linum usitatissimum). Por lo tanto, el valor agregado será la mezcla natural envasada, bebible y de fácil digestión. Como producto básico satisface las necesidades primarias de sed y se le puede acompañar con galletas, tostadas, comidas, entre otros alimentos. Como producto físico, el envase será de vidrio de $300 \mathrm{ml}$ neto, se utilizarán tapas de twist off con facilidad de cierre y abertura con fracción de vuelta y su comercialización será por unidad. En cuanto a la etiqueta, estará regulada según la NTP 209.038 "Alimentos envasados y etiquetados" y el Reglamento sobre Vigilancia y Control Sanitario de Alimentos y Bebidas aprobado por DS 007-98-SA. Como producto aumentado, se tiene un producto diferenciado, cuya combinación aún no existe en el mercado, como la linaza y el tumbo. Además, se presentará en una red social o blog la información nutricional del tumbo y la linaza, recetas de comidas para acompañar con la bebida y dietas sugeridas.

El target market que abarcará el estudio es Lima Metropolitana, dirigido para consumidores que valoran los productos saludables y que previenen distintos malestares. Se encontró un atractivo mercado en los niveles socioeconómicos A y B, orientado a un público de 18 a 60 años.

Por otro lado, las principales materias primas son el tumbo andino (Passiflora mollissima H. B. K), la linaza (Linum usitatissimum) y también insumos como el agua tratada y el edulcorante natural o estevia.

El tumbo es una especie de fruta nativa que se ubica especialmente en la sierra media y parte de la alta, posee gran valor nutritivo debido a 


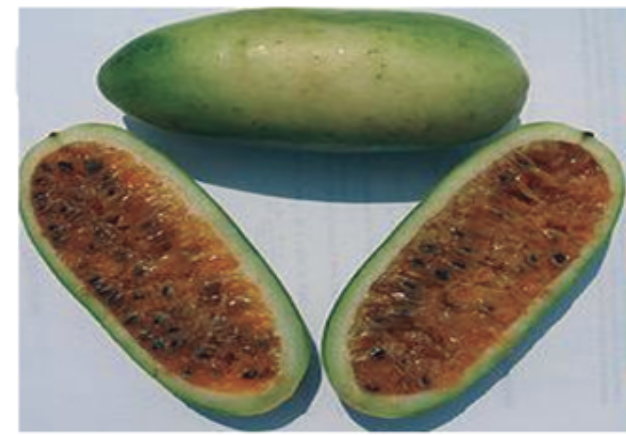

Ecotipo agrio

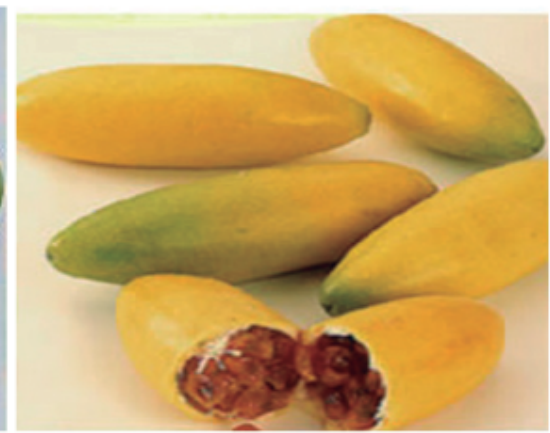

Ecotipo dulce

Figura 1. Frutos de los ecotipos de tumbo Elaboración propia

su alto contenido de sustancias biológicamente activas como las vitaminas C, A y enzimas diversas. Además, existen dos ecotipos: uno de ellos es el ecotipo dulce, que sobresale por la cantidad de pulpa dulce en su interior, y el ecotipo agrio, cuya diferencia se ve en la longitud del fruto, el valor del $\mathrm{pH}$ más alto y cuenta con un mayor número de semillas en el fruto, el de mejor propagación sexual (semillas), y buenas características para su transporte y almacenamiento. En la figura 1 se observan los dos ecotipos; sin embargo, debido a la heterogeneidad del suelo, se utilizarán ambos, pero con orientación a los agricultores se buscará el ecotipo agrio.

En cuanto al clima, esta planta es de zonas altas y frías, con mayor éxito en los valles interandinos, pues las temperaturas varían entre 3,8 y $24^{\circ} \mathrm{C}$. La época más apropiada para el desarrollo de la actividad agrícola son las estaciones de primavera y verano (Servicio Nacional de Meteorología e Hidrología-Senamhi, Tacna).

Este fruto aporta beneficios significativos para la salud, como la vitamina $\mathrm{C}$, y es un agente antioxidante que incrementa la absorción de hierro a nivel gástrico. Es bueno para evitar y tratar la anemia, nutre la piel, elimina arrugas y recupera su elasticidad, además de revitalizar el organismo sin producir un aumento de peso. También evita los cálculos renales, malestares urinarios y dolores estomacales, disminuye el deterioro de la edad, pues es conocido como el fruto del antienvejecimiento. En su composición se ha descubierto serotonina, un potente neurotransmisor necesario para el buen estado del sistema nervioso y cuya deficiencia es responsable de patologías como la depresión, ciertos 
tipos de obesidad, comportamientos obsesivos, insomnio y migrañas. Otras investigaciones señalan que los compuestos bioactivos y la capacidad antioxidante que posee el fruto podrían estar relacionados con la disminución del riesgo de enfermedades degenerativas como el cáncer, cataratas o disfunciones del cerebro.

En la actualidad, el tumbo todavía es una fruta relativamente desconocida en el mercado peruano. En cuanto a su producción, no presenta datos actualizados. Sin embargo, se visitaron algunos mercados locales ubicados en la región Junín (Huancayo y Tarma) y se observaron diversos productores de tumbo, que ofrecían el fruto en jabas durante su temporada alta. Como se observa en la figura 2 , también se encontró en el mercado de Surquillo.

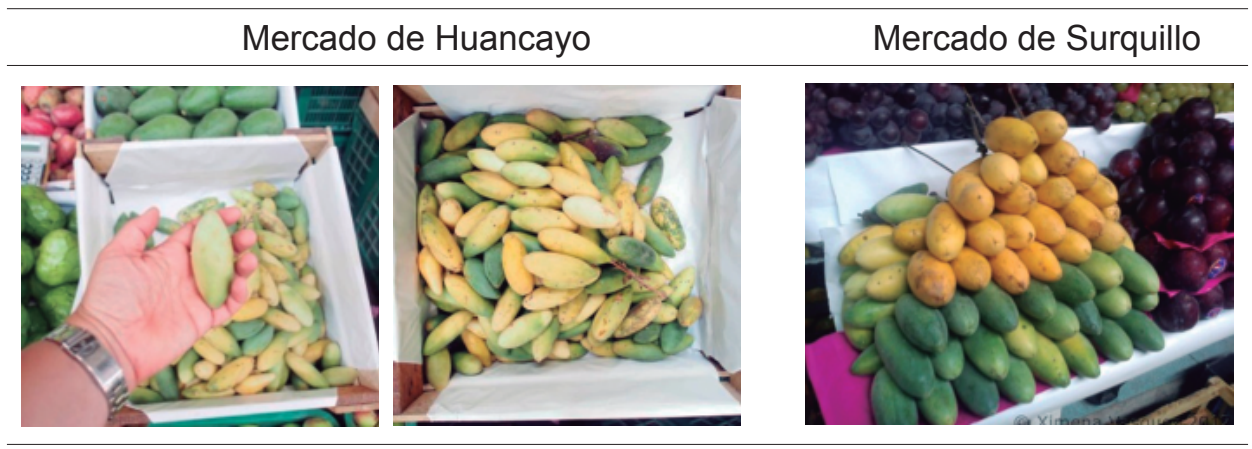

Figura 2. Proveedores de tumbo Elaboración propia

Para el proyecto se capacitará al productor con técnicas de propagación y mejoramiento del cultivo realizadas por INIA ${ }^{1}$ e IICA ${ }^{2}$. Se designará a una persona para gestionar y preparar el lote que será enviado a la planta semanalmente.

En cuanto a la linaza, proviene de Canadá y Estados Unidos, aunque se cree que esta planta es originaria de Egipto. Lo importante en ella es la semilla de lino, a la que se conoce como linaza. Para la elaboración del producto, utilizaremos esta materia prima con grandes aportes nutricionales para la salud, debido a los componentes vitamínicos que posee (véase tabla 1 ).

1 Véase Instituto de Investigación Agropecuaria (2015).

2 Véase Instituto de Investigación Científica Agropecuaria (s.f.). 
Tabla 1

Contenido vitamínico de la linaza

\begin{tabular}{lcc}
\hline Soluble en agua & mg / $\mathbf{1 0 0 ~ g ~}$ & $\begin{array}{c}\text { mg/cuchda. } \\
\text { Linaza molida }\end{array}$ \\
\hline Ácido ascórbico / vitamina C & 0,50 & 0,04 \\
Tiamina / vitamina B 1 & 0,53 & 0,04 \\
Riboflavina/ vitamina B2 & 0,23 & 0,02 \\
Niacina / ácido nicótico & 3,21 & 0,26 \\
Piridoxina / vitamina B & 0,61 & 0,05 \\
Ácido pantoténico & 0,57 & 0,05 \\
& mcg / 100 g & mcg / 100 g \\
Ácido fólico & 112 & 9,0 \\
Biotina & 6 & 0,5 \\
\hline Soluble en grasa & mg / kg en aceite & mg / cuchda. en aceite \\
\hline Carotenos & No detectados & No detectados \\
Vitamina E & & \\
$\quad$ Alfa-tocoferol & 7 & 0,10 \\
Delta-tocoferol & 10 & 0,14 \\
Gamma-tocoferol & 552 & 7,73 \\
Vitamina K & & mcg / cuchda. de linaza molida \\
\hline
\end{tabular}

Nota:

a Muestra compuesta de linaza entera.

b Valores de tocoferol representan el promedio de cuatro variedades. Las siguientes formas de vitamina $\mathrm{E}$ no fueron detectadas: beta-tocoferol y alfa-, delta-, y gamatocotrienol.

C Como filoquinona.

Fuente: Flax Council of Canada (2005)

Como menciona el Centro Nacional de Medicina Alternativa, no hay estudios concluyentes sobre muchos de los beneficios de la linaza, pero se conocen los componentes nutricionales como la fibra y el omega 3 , que favorece a las personas que buscan reducir tallas y medidas. Esto se debe a que acelera el metabolismo y con ello la pérdida de células grasas. Para el sistema digestivo, previene o cura el cáncer de colon, además de ser ideal contra la gastritis, estreñimiento y acidez estomacal, lubricando y regenerando la flora intestinal. También es ideal contra la diabetes porque su consumo regular favorece el control de los niveles de azúcar en la sangre. 


\section{LOCALIZACIÓN DE LA PLANTA}

El análisis de los factores de localización es fundamental para la decisión de la ubicación de la planta industrial. Es por esto que para el desarrollo del trabajo de investigación se tomaron los siguientes factores de macrolocalización relevantes como la proximidad a la materia prima, proximidad al mercado, disponibilidad de mano de obra, disponibilidad de energía eléctrica, disponibilidad de agua, transporte y flete, terreno, clima, eliminación de desechos y condiciones de vida. Al utilizar el método de ranking de factores para los departamentos de Lima, La Libertad, Junín y Huancavelica, se obtuvo que la región Lima es la mejor opción, dado que tuvo el mejor puntaje con respecto a las demás alternativas. Por lo tanto, la instalación de la planta se hará en Lima. Por otro lado, en la microlocalización la alternativa de mayor puntaje en el ranking de factores dio como resultado el distrito de Lurín.

\section{TAMAÑO DE LA PLANTA}

El tamaño de planta está determinado por la capacidad de producción y el tamaño de mercado, pero como el tamaño de la tecnología definido por la marmita (6655 unidades de bebida/día) excede al mercado (4363 unidades/día), se decide que el tamaño de planta será igual al tamaño del mercado. No obstante, la disponibilidad del recurso en bajas temporadas podría limitar la demanda (véase tabla 2). Por ello, se capacitará al productor con técnicas de cultivo para elevar la producción o se optará por importar la fruta de los principales países productores de tumbo como Colombia, Brasil, Ecuador, Venezuela o Bolivia.

Tabla 2

Selección del tamaño de planta

\begin{tabular}{lc}
\hline \multicolumn{1}{c}{ Factor } & $\begin{array}{c}\text { Tamaño de planta } \\
\text { (unidad de bebida/ día) }\end{array}$ \\
\hline T. mercado & 4363 \\
T. disponibilidad de recurso & 11601 (baja) y 29302 (alta) \\
T. tecnología & 6655 \\
T. punto de equilibrio (618 155 unidades) & 1981 \\
\hline
\end{tabular}

Elaboración propia 


\section{INGENIERÍA DEL PROYECTO}

Se presenta el diseño tentativo del producto "Bebida natural de tumbo con linaza" en la figura 3 .

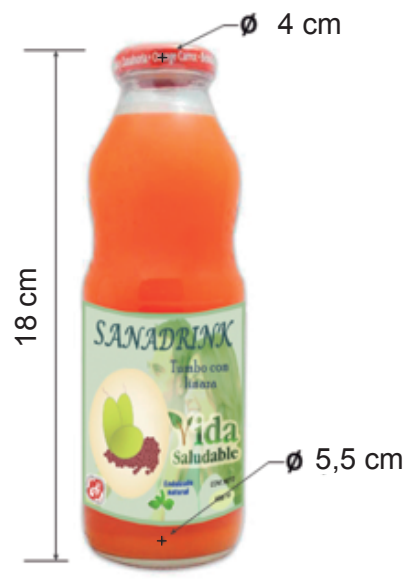

Figura 3. Diseño y medidas del producto Elaboración propia

Tabla 3

Especificaciones de la bebida de tumbo con linaza

\begin{tabular}{|c|c|}
\hline \multicolumn{2}{|c|}{ Descripción del producto y uso presunto } \\
\hline Nombre & Bebida de tumbo con linaza \\
\hline Descripción & Bebida de fruta con ingredientes autorizados \\
\hline Composición & Pulpa de tumbo, linaza, estevia, ácido cítrico, agua \\
\hline Características sensoriales & $\begin{array}{l}\text { Color y sabor de la pulpa de fruta } \\
\text { Consistencia líquida }\end{array}$ \\
\hline $\begin{array}{l}\text { Características fisicoquímicas } \\
\text { y microbiológicas }\end{array}$ & $\begin{array}{l}\mathrm{pH}<4,5 \text {, sabor agridulce, } 5 \% \text { de sólidos solubles } \\
\text { mínimo, olor a fruta de la que procede, exenta de } \\
\text { parásitos, mohos, levaduras y microorganismos } \\
\text { patógenos (NTP 203.110) }\end{array}$ \\
\hline $\begin{array}{l}\text { Forma de uso y consumidores } \\
\text { potenciales }\end{array}$ & Acompañante de comidas \\
\hline $\begin{array}{l}\text { Empaque, etiquetado } \\
\text { y presentaciones }\end{array}$ & En envases de vidrio, en presentaciones de $300 \mathrm{ml}$ \\
\hline Vida útil esperada & Seis meses sin destapar \\
\hline
\end{tabular}

Elaboración propia 
Los resultados de las pruebas de laboratorio van acorde con las normas y requisitos técnicos para la fabricación de una bebida de fruta. En la tabla 3 se presenta la descripción, composición y uso presunto del producto.

La industria de bebidas de fruta presenta diversos procesos de fabricación y utiliza distintos tipos de tecnologías como manual, semiautomatizada o automatizada.

Hoy en día, la tecnología semiautomática y automatizada ha reemplazado al proceso manual, a pesar de su elevado costo, con lo cual ha logrado maximizar los beneficios de la empresa. Por ejemplo, las fábricas de la corporación Lindley, corporación Backus o Ajegroup hacen uso de este tipo de tecnología. A continuación se mostrará el tipo de tecnología seleccionada en cada proceso.

Tabla 4

Selección de la tecnología

\begin{tabular}{lll}
\hline Operación & Tecnología elegida & \multicolumn{1}{c}{ Sustentación } \\
\hline Lavado & Lavadero industrial & $\begin{array}{l}\text { No es necesario contar con una máquina } \\
\text { especializada, dado que la fruta es muy de- } \\
\text { licada y requiere manipulación adecuada. } \\
\text { lgual para el lavado de linaza. }\end{array}$ \\
\hline Escaldado & Tanque de escaldado & $\begin{array}{l}\text { Se eligió el tanque escaldado, el cual } \\
\text { trabajará por inmersión (en donde se } \\
\text { introduce la fruta). Se deberá controlar la } \\
\text { temperatura y el tiempo. }\end{array}$ \\
\hline Pulpeado & Despulpadora & $\begin{array}{l}\text { Máquina de buen rendimiento; posee la ca- } \\
\text { pacidad adecuada para la operación de } \\
\text { despulpado. }\end{array}$ \\
\hline horizontal & Marmita con agitador & $\begin{array}{l}\text { Esta tecnología es la más apropiada, ya que } \\
\text { el agitador de paletas ayudará a incrementar } \\
\text { el rendimiento del producto. }\end{array}$ \\
\hline Filtrado & Tamiz vibratorio & $\begin{array}{l}\text { Presenta una alta compactación, que } \\
\text { ayudará a separar el gel de la linaza de las } \\
\text { semillas. }\end{array}$ \\
\hline Mezclado & Mezclador de & $\begin{array}{l}\text { Las paletas verticales e inclinadas ayudarán } \\
\text { a que la mezcla sea más homogénea. }\end{array}$ \\
\hline & paletas &
\end{tabular}




\begin{tabular}{lll}
\hline Operación & Tecnología elegida & \multicolumn{1}{c}{ Sustentación } \\
\hline Pasteurizado & Pasteurizador & $\begin{array}{l}\text { Se utilizará el método HTST, dado que } \\
\text { expone al alimento a grandes temperaturas } \\
\text { en un periodo breve. }\end{array}$ \\
\hline Envasado & Semiautomática & $\begin{array}{l}\text { Presenta la capacidad requerida por nuestra } \\
\text { línea de producción. }\end{array}$ \\
\hline Tapado & Semiautomática & $\begin{array}{l}\text { Es de menor costo y de fácil manejo para el } \\
\text { operario. }\end{array}$ \\
\hline $\begin{array}{l}\text { Etiquetado y } \\
\text { codificado }\end{array}$ & Automático & $\begin{array}{l}\text { Se requiere el pegado y codificado con } \\
\text { chorros de tinta automático uniforme. }\end{array}$ \\
\hline Enfriado & Túnel de enfriamiento & $\begin{array}{l}\text { Enfriamiento rápido con el uso eficiente del } \\
\text { agua, ya que retiene y recircula el agua. } \\
\text { Realiza el sellado al vacío debido al choque } \\
\text { térmico. }\end{array}$ \\
\hline $\begin{array}{l}\text { Esterilizador } \\
\text { UV }\end{array}$ & Automático & $\begin{array}{l}\text { Es ideal para el proceso, además de su bajo } \\
\text { costo de mantenimiento. }\end{array}$ \\
\hline $\begin{array}{l}\text { del agua } \\
\text { Esto }\end{array}$ & Esterilización UV & $\begin{array}{l}\text { Posee bajo costo de mantenimiento en } \\
\text { su instalación, además de su eficiente } \\
\text { tratamiento. }\end{array}$ \\
\hline
\end{tabular}

Elaboración propia

Para la elaboración del proceso se tomó como base el proceso presentado en la tesis "Estudio de prefactibilidad para la implementación de una planta de elaboración de bebida de papaya (Carica papaya) con linaza (Linum usitatissimum)" (Pardo Delgado y Urquizo Baldárrago, 2014), pues su composición y características son similares al producto propuesto. En la figura 4 se muestra el diagrama de operaciones del proceso para la elaboración de una bebida a base de tumbo andino con linaza en presentación de six pack de $300 \mathrm{ml}$ c/u.

El proceso se describe a continuación.

a) Inspección y seleccionado de la materia prima

Inicia con el arribo de los camiones. Se recibirá el tumbo en cajas de los proveedores de Junín, y la linaza de los principales centros de acopio, como el mercado Mayorista de Lima. Los camiones descargarán las materias primas en la zona de descarga cerca de los 
almacenes, donde el tumbo y la linaza serán transportados desde el almacén de materias primas, por medio de carros de estantería, hasta la estación de selección.

b) Pesado de la materia prima

Luego de la selección e inspección, un operario se encargará de pesar los tumbos y la linaza, con el fin de determinar la cantidad requerida que entrará al proceso productivo. Asimismo, se podrá controlar el rendimiento de la materia prima.

c) Lavado

La materia prima será trasladada hacia la estación de lavado, donde la fruta será lavada de manera manual en un lavadero industrial con una solución de hipoclorito de sodio: $3 \mathrm{ml}$ de solución de hipoclorito de sodio al $3 \%$ por cada 100 litros de agua ${ }^{3}$. En el caso de la linaza, esta será lavada en otro lavadero industrial con un colador y chorros de agua a presión constante.

d) Pelado

El tumbo, exento de materias extrañas, será llevado por medio de carros de estantería a la estación de pelado. Esta operación se realizará de manera manual en una mesa de trabajo. En esta operación se pierde aproximadamente el $17,68 \%$, dato que se obtuvo en las pruebas de laboratorio.

e) Escaldado

Mediante fuentes de acero inoxidable, la fruta sin cáscara será transportada a la zona de escaldado, donde un operario introducirá el tumbo en un tanque de escaldado. A su vez, se tendrá que mantener un control sobre los parámetros de temperatura que están en el rango de $70{ }^{\circ} \mathrm{C}$ a $75{ }^{\circ} \mathrm{C}$ durante dos minutos, para evitar peligros microbiológicos y bacterianos.

f) Pulpeado

Con la máquina pulpeadora se obtendrá la pulpa de tumbo exenta de pepas; en esta operación se tendrá desperdicios (las semillas o pepas). A nivel industrial, la operación se realizará en despulpadoras industriales. La pulpa del tumbo será trasladada por medio de tuberías de acero inoxidable a un tanque de almacenamiento temporal en la zona de mezclado.

3 Véase Pardo Delgado y Urquizo Baldárrago (2014). 


\section{g) Cocción en marmita}

Paralelamente, luego de que la linaza haya pasado por los procesos de pesado, seleccionado y lavado, será llevada a una marmita, donde se hierve agua con la linaza que previamente ha sido pesada en la siguiente proporción: por cada 1,25 kilos de linaza ingresan 50,1 litros de agua. La cocción se realizará aproximadamente por 10 minutos (Pardo Delgado y Urquizo Baldarrágo, 2014). De esta manera, se obtiene el gel de linaza, el cual será mezclado con la pulpa en las siguientes operaciones.

h) Filtrado

En el proceso de cocción se obtiene el gel de linaza. Este, junto con las semillas, se trasladará por un túnel al cernidor vibratorio de granulometría apropiada, que consiste en separar el gel de linaza de las semillas, las que se depositarán en dos tanques. Luego, el gel de linaza se trasladará por medio de tuberías de acero inoxidable al tanque de almacenamiento temporal en la zona de mezclado, mientras que las semillas de linaza se trasladarán a la operación de cocción, en la que se realizará un reproceso y luego será retirada. En esta estación se pierde alrededor del 23,05\% de peso.

i) Mezclado

Una vez que la pulpa y el gel de linaza estén en los tanques de almacenamiento temporal, se dosificará y se mezclará. Además, se adicionarán ácido cítrico, estevia (previamente pesada) y el agua tratada será dosificada. De la máquina mezcladora se obtendrá un líquido homogéneo y uniforme.

j) Pasteurizado

Luego de obtener una mezcla homogénea, pasará a la máquina pasteurizadora por medio de tuberías de acero inoxidable. En el caso de alimentos líquidos, la temperatura tendría que situarse sobre los $70{ }^{\circ} \mathrm{C}$ y $85^{\circ} \mathrm{C}$, de 12 a 15 minutos.

k) Envasado y tapado

Una vez pasteurizada, será enviada por tuberías de acero inoxidable al proceso de envasado. Sin embargo, los envases pasarán por un proceso de esterilización, en el que un operario coloca los envases en la máquina de enjuague. Luego, por medio de una faja transportadora, serán enviadas a la máquina envasadora. Una vez que se encuentren en esta máquina, un operario realizará la dosificación en 
caliente $\left(70\right.$ a $\left.85^{\circ} \mathrm{C}\right)$ con 300 mililitros de bebida de tumbo con linaza por cada envase, y rápidamente serán tapados.

1) Enfriado

Las bebidas envasadas y tapadas pasarán a ser enfriadas por medio de un túnel de enfriamiento con chorros de agua fría y el choque térmico realizará el sellado al vacío, dándole al producto un sellado único y eficiente.

m) Etiquetado

Para el proceso de etiquetado se contará con una empresa que preste el servicio de elaboración de etiquetas, las que serán añadidas a nuestro producto por medio de una máquina etiquetadora. Es importante destacar que las etiquetas deben cumplir con los requisitos establecidos por el Instituto Nacional de Defensa de la Competencia y de la Protección Intelectual (Indecopi, 2008).

n) Codificado

Una vez etiquetado el producto, pasa por el proceso de codificado, en el que se imprime sobre la tapa la fecha de vencimiento y el número de lote.

o) Almacenado

Los six pack de bebida de tumbo se almacenarán en una caja que contendrá en total 24 botellas.

Para determinar la capacidad instalada, se tomó en consideración la capacidad de los equipos y su utilización de acuerdo a los turnos de trabajo que se han definido: 8 horas diarias, 1 turno por día, 6 días a la semana, 52 semanas al año. De esta manera, se halla la capacidad instalada bajo la metodología de un sistema de producción en línea a partir del concepto de cuello de botella y otros elementos que intervienen para determinar la capacidad, como el factor de utilización, que considera la desviación que existe entre las horas reales y las horas productivas; y el factor de eficiencia, que considera la desviación que existe entre las horas estándar y las horas producidas. El cuello de botella es el proceso de cocción determinada por la capacidad de la marmita. 


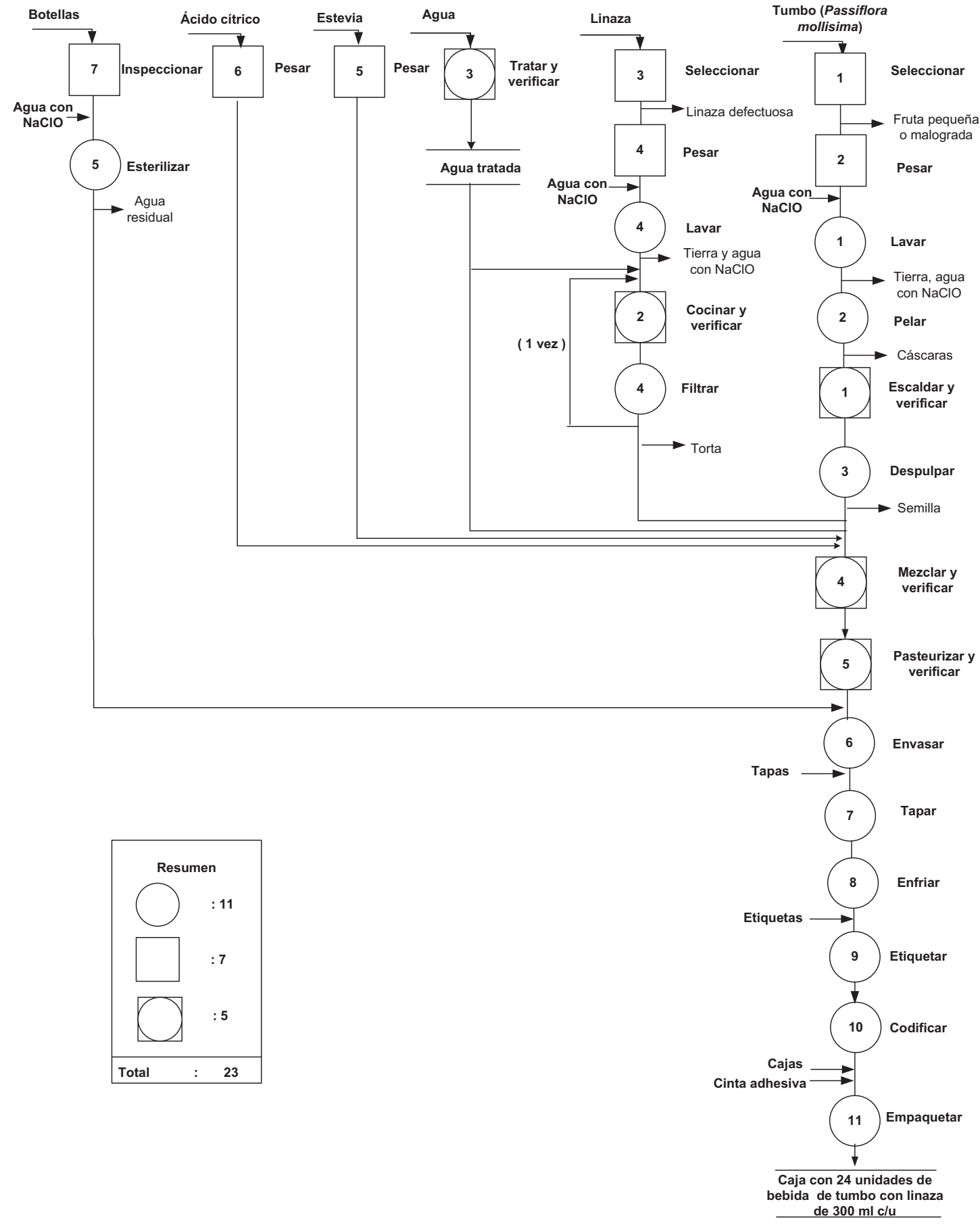

Figura 4. Diagrama de operaciones del proceso para la elaboración de bebida del tumbo andino con linaza $300 \mathrm{ml}$

Elaboración propia 


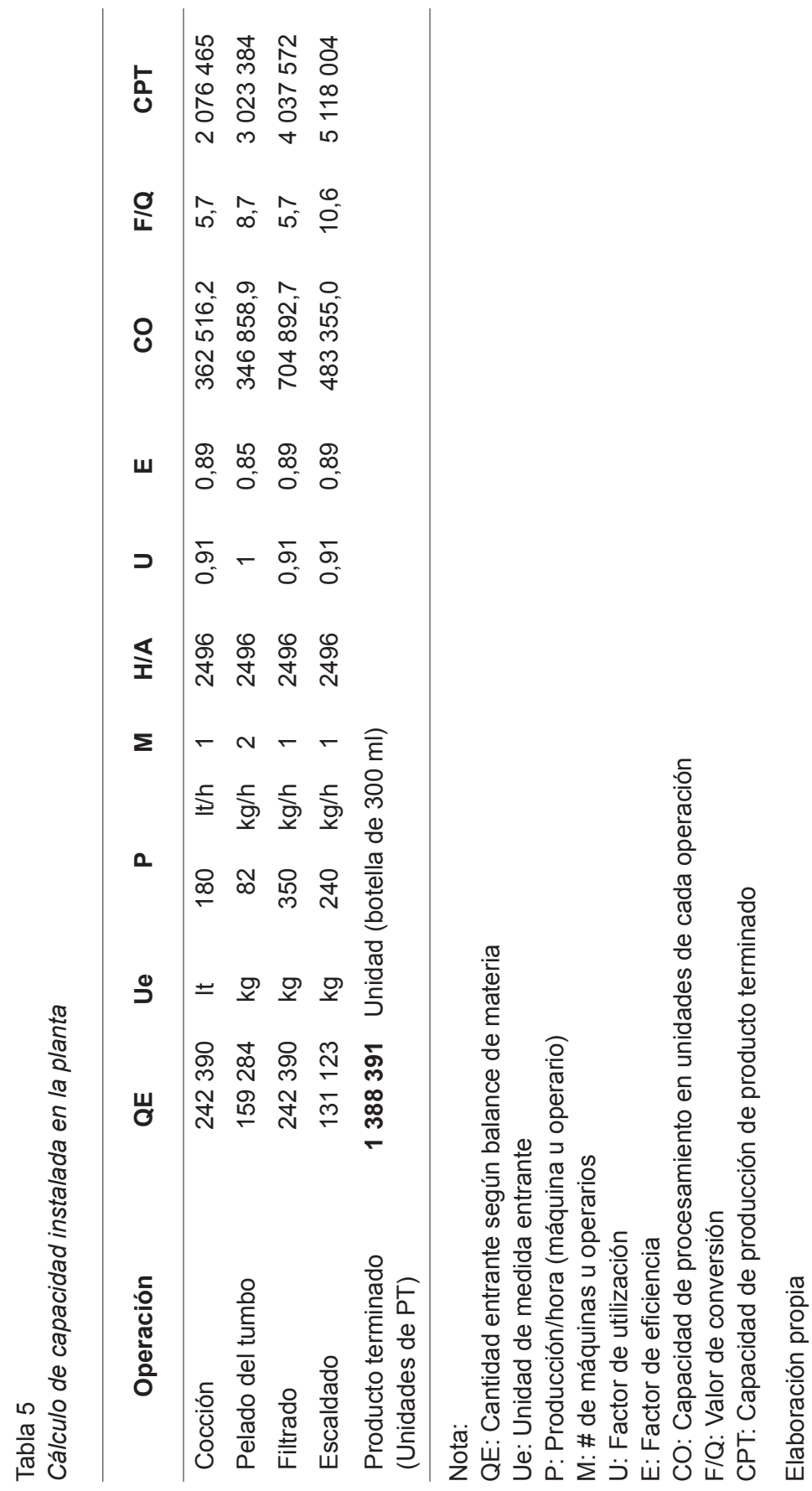


Además, se implementará el programa de Análisis de Peligros y Puntos Críticos de Control (HACCP), cuyo requisito es obligatorio para la obtención de la habilitación sanitaria respectiva. Para lograr la seguridad alimentaria es necesario contar con buenas prácticas de manufactura (BPM), tener presente los procedimientos operacionales estandarizados de sanitización (POES), como la limpieza y desinfección de las instalaciones, equipos, indumentaria, manos de los operarios, entre otros accesorios involucrados en el área de producción.

En cuanto al estudio del impacto ambiental, la evaluación con la matriz de Leopold determinó que el proyecto durante sus etapas generará un impacto negativo moderado, clasificándolo en la categoría II. Esto quiere decir que para obtener la certificación ambiental se requiere el estudio del impacto ambiental semidetallado. Paralelamente, se debe tener en cuenta el Reglamento de Seguridad y Salud en el Trabajo y el DS N. ${ }^{\circ} 009-2005-\mathrm{TR}$ y su modificatoria DS N. ${ }^{\circ} 007-2007-\mathrm{TR}$ para un ambiente seguro de trabajo.

\section{DISPOSICIÓN DE LA PLANTA}

Se contará con las siguientes áreas, como se muestra en la siguiente tabla.

Tabla 6

Áreas comprendidas en la planta de producción

\begin{tabular}{ll}
\hline \multicolumn{1}{c}{ Área } & \multicolumn{1}{c}{ Descripción } \\
\hline $\begin{array}{l}\text { Almacén de materia } \\
\text { prima }\end{array}$ & $\begin{array}{l}\text { En esta área se almacenarán las jabas de fruta fresca } \\
\text { (tumbo), los sacos de las semillas de linaza, los sacos de } \\
\text { estevia y los sacos de ácido cítrico. }\end{array}$ \\
\hline
\end{tabular}

Almacén de insumos

Se almacenarán las botellas, etiquetas, tapas, cajas y rollos del film retráctil.

Área de producción Área en que se desarrollará el proceso productivo. Debe estar ubicada cerca a los almacenes.

Almacén de producto terminado
Área para destinar los sixpack que serán previamente llenados en cajas listas para su despacho. 
(continuación)

\begin{tabular}{ll}
\hline \multicolumn{1}{c}{ Área } & \multicolumn{1}{c}{ Descripción } \\
\hline $\begin{array}{l}\text { Vestuarios y baño del } \\
\text { personal }\end{array}$ & $\begin{array}{l}\text { Según la OSHA, se debería colocar un mínimo de dos } \\
\text { baños (16 a } 35 \text { personas). Sin embargo, para comodidad del } \\
\text { operario y del personal administrativo, se contará con dos } \\
\text { servicios higiénicos independientes para cada género. }\end{array}$ \\
\hline
\end{tabular}

Oficina de la gerencia general

$\begin{array}{ll}\text { Oficina administrativa } & \text { Área amplia destinada para los jefes intermedios, como los } \\ \text { de logística, marketing, contabilidad, finanzas y recursos } \\ \text { humanos. }\end{array}$

Área administrativa, donde se ubicará el máximo líder, que además debe contar con un espacio para la sala de reuniones.
Oficina de producción Área de administración cercana al área de producción para la supervisión respectiva.

Oficina de calidad

En esta área se realizan pruebas de laboratorio, como las pruebas de dulzor (grados brix), nivel de acidez, entre otras, para garantizar la calidad del producto final.

\begin{tabular}{ll}
$\begin{array}{l}\text { Servicios higiénicos del } \\
\text { personal administrativo }\end{array}$ & Se debe contar con dos espacios para cada género. \\
\hline Patio de maniobras & $\begin{array}{l}\text { Área que será empleada para el tránsito del montacargas y el } \\
\text { arribo de los camiones. }\end{array}$ \\
\hline Comedor & $\begin{array}{l}\text { Ambiente donde el personal puede tomar su refrigerio con } \\
\text { sillas y mesas adecuadas. }\end{array}$
\end{tabular}

Área de tratamiento de agua

Área de mantenimiento

Zona del compresor

En esta área habrá una cisterna ubicada en el subsuelo, y en el suelo se tendrá el tanque de agua, la bomba de agua y el esterilizador UV.

Área destinada para trasladar los equipos que requieren reparaciones o cambios de pieza, y también abastecerá de herramientas.

Área habilitada para la máquina generadora de aire comprimido. Se ubicará cerca de la zona de producción para alimentar a ciertas máquinas.

Área de sanitización

Área adecuada para el ingreso al proceso productivo, donde se desinfectará la indumentaria del operario con el fin de evitar el ingreso de agentes patógenos. 
Por otro lado, para el dimensionamiento de las áreas de la planta se utilizó el método de Guerchet, para obtener el área de las superficies de distribución. El dimensionamiento del almacén se calculó en función al tamaño de la demanda, y para las áreas administrativas fueron tomadas de Sule (2012).

A continuación, se muestra las dimensiones requeridas para la planta. Sin embargo, estas dimensiones pueden variar de acuerdo a las características del terreno.

Tabla 7

Área mínima requerida para el proyecto

\begin{tabular}{lcc}
\hline \multicolumn{1}{c}{ Área } & $\begin{array}{c}\text { Dimensión } \\
(\mathbf{m})\end{array}$ & $\mathbf{m}^{2}$ \\
\hline Gerencia general & $6 \times 5$ & 30 \\
Administrativa & $15 \times 5$ & 75 \\
Secretaría & $4 \times 3$ & 12 \\
Servicio higiénico administración) & $3 \times 2,5$ & 7,5 \\
Comedor & $5,5 \times 4$ & 22 \\
Jefe de producción & $6 \times 3$ & 18 \\
Laboratorio de calidad & $6 \times 3$ & 18 \\
Mantenimiento & $6 \times 3$ & 18 \\
Producción & - & 167 \\
Sanitización & $3,5 \times 3$ & 10,5 \\
Servicio higiénico (auxiliar) & $(2,5 \times 1,5)$ & 3,75 \\
Caldero & $3 \times 3,5$ & 10,5 \\
Tratamiento de agua & $5 \times 3$ & 15 \\
Almacén de MP & $6 \times 5$ & 30 \\
Servicio higiénico y vestuario de operario & $4 \times 2(2)$ & 16 \\
Almacén de PT & $16 \times 4,5$ & 72 \\
Almacén de insumos & $7 \times 4$ & 28 \\
Compresor & $3 \times 3,5$ & 10,5 \\
Patio de maniobras & $10 \times 7$ & 70 \\
Tránsito interno & - & - \\
Vigilancia & $2,5 \times 2,5$ & 6,25 \\
\hline & 640,29 & \\
\hline & & \\
\hline & Total área mínima & \\
& &
\end{tabular}

Elaboración propia

En la siguiente figura se muestra el dimensionamiento y la distribución de las áreas. 


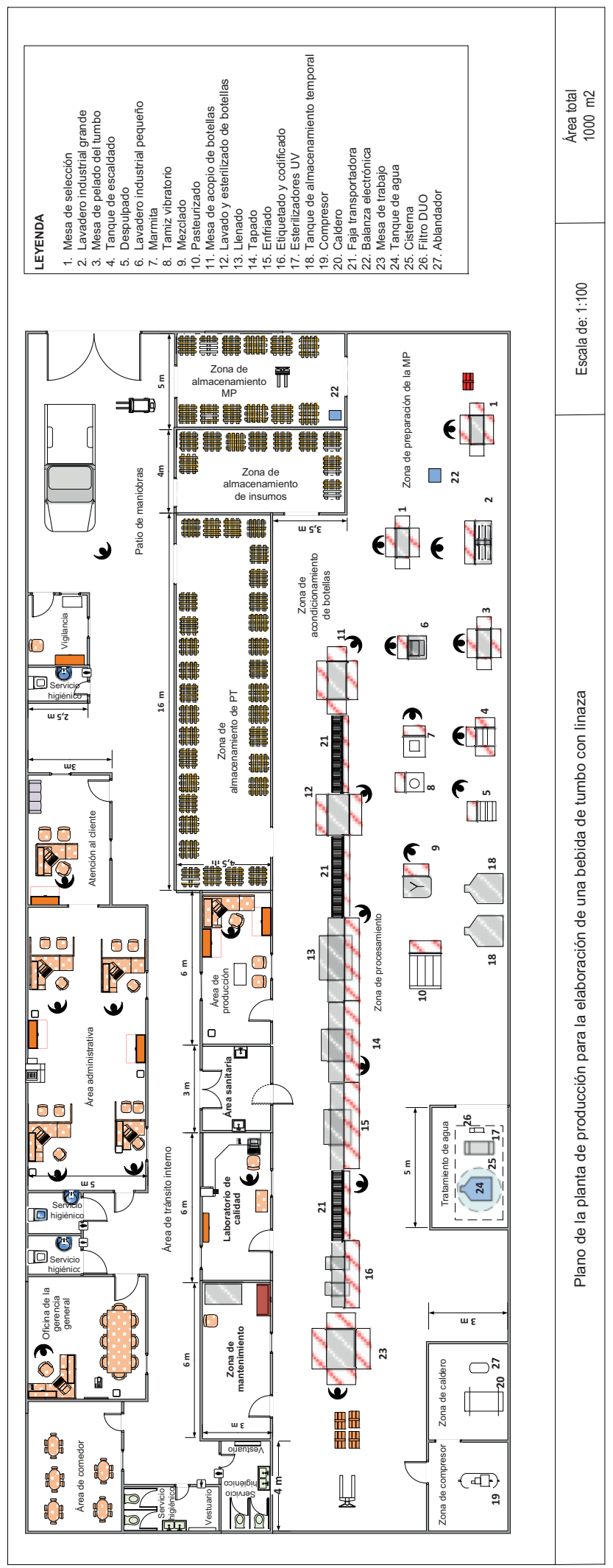

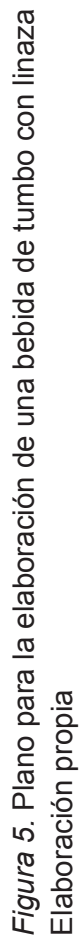




\section{ORGANIZACIÓN Y ADMINISTRACIÓN}

Dentro de los sistemas de organización de empresas existen tres modelos. Uno de ellos es el modelo lineal, que es el más antiguo y simple; el siguiente es el funcional, en el que existen especialistas dedicados a determinados trabajos y cada especialista está bajo el mando de un jefe; el tercer modelo es el mixto o matricial, especial para empresas que poseen actividades agrupadas por funciones y por proyectos. El proyecto se adecúa al modelo lineal, debido al requerimiento mínimo de personal administrativo, recomendado para Mypes. A continuación se presenta la estructura organizacional.

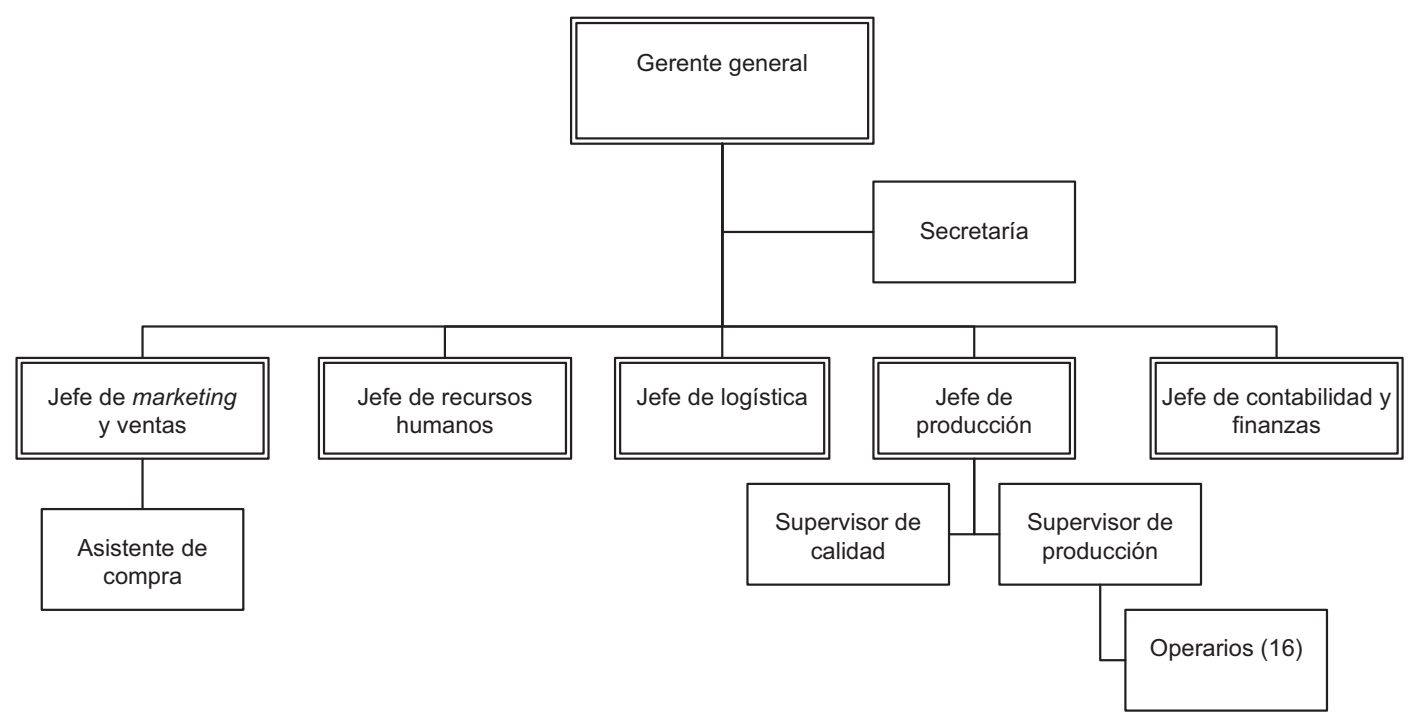

Figura 6. Organigrama de la empresa

Elaboración propia 


\section{ASPECTOS ECONÓMICOS Y FINANCIEROS}

A continuación se detalla la inversión del proyecto.

Tabla 8

Resumen de la inversión en activos

\begin{tabular}{lrr}
\hline Activos tangibles & Monto (S/) & Total (S/) \\
\hline Inversión en máquinas & 194340 & \\
Inversión en equipos de transporte & 14530 & \\
Equipos e instrumentos de planta & 8358 & 1317856 \\
Inversión en terreno & 625195 & \\
Obras civiles & 373954 & \\
Muebles, enceres y equipos de oficina & 38724 & \\
Imprevistos & 62755 & \\
Activos intangibles & 10635 & \\
Estudio de preinversión & 18220 & $\mathbf{3 4 4 0 5}$ \\
Gastos de gestión & 3912 & 1638 \\
Gastos de organización y constitución de la empresa & & \\
Contingencias (5 \%) & & \\
Total activos tangibles e intangibles (S/) & & \\
\hline
\end{tabular}

Elaboración propia

Tabla 9

Inversión del proyecto

\begin{tabular}{lr}
\hline Rubro & Monto (S/) \\
\hline Act. tangible & 1317856 \\
Act. intangible & 34405 \\
Capital de trabajo & 501949 \\
Inversión total & $\mathbf{1 8 5 4 2 1 0}$ \\
\hline
\end{tabular}

Elaboración propia 
Para iniciar el proyecto, se decide empezar con un ratio de deuda/inversión de 0,4. Por ello, la empresa tendría la estructura que se muestra en la siguiente figura.

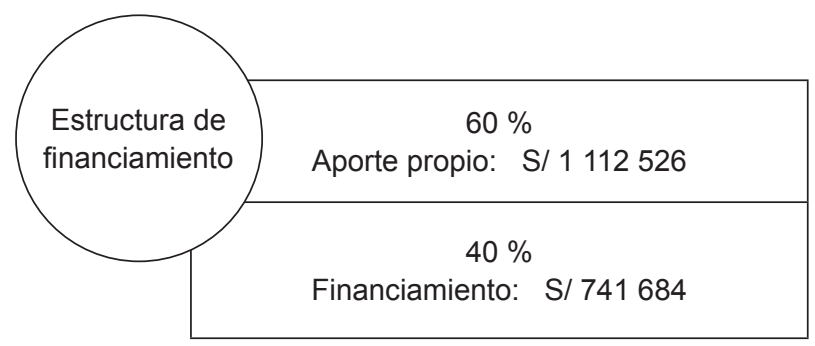

Figura 7. Estructura de financiamiento

Elaboración propia

\section{EVALUACIÓN ECONÓMICA Y FINANCIERA DEL PROYECTO}

En la evaluación económica y financiera del proyecto se obtuvo los siguientes resultados, como se muestra a continuación:

Tabla 10

Indicadores económico-financieros

\begin{tabular}{cc}
\hline \multicolumn{2}{c}{ Indicadores económicos } \\
\hline VAN & 162084 \\
TIR & $28,30 \%$ \\
B/C & 1,09 \\
P. R. & 4,76 \\
\hline
\end{tabular}

\begin{tabular}{cc}
\hline \multicolumn{2}{c}{ Indicadores financieros } \\
\hline VAN & 325140 \\
TIR & $35,04 \%$ \\
B/C & 1,4 \\
P. R. & 4,44 \\
\hline
\end{tabular}

Elaboración propia

\section{CONCLUSIONES}

- Con respecto al perfil del consumidor, se concluyó que el mercado objetivo está constituido por personas que se encuentren entre los 18 y los 60 años, pertenecientes a los niveles socioeconómicos A y B, cuyos hábitos de consumo son de preferencia natural y saludable. La 
demanda inicial para el proyecto será de 1079319 botellas de 300 mililitros.

- Del análisis técnico se determinó que el mejor lugar para ubicar la planta se encuentra en el departamento de Lima, en la zona industrial del distrito de Lurín.

- El tamaño máximo de la planta está determinado por el mercado, con una producción de 4363 envases por día. Por otro lado, el tamaño mínimo de la planta está determinado por el punto de equilibrio, con 618155 envases anuales. Además, la capacidad de la planta está determinada por la marmita (cuello de botella), cuya producción es de 6655 envases por día.

- El monto de inversión total estimado es de S/ 1854 211, con un financiamiento del $40 \%$, con un costo de deuda promedio en el mercado de $20,61 \%$ y el restante $60 \%$ por parte de los accionistas. El capital de trabajo asciende a S/ 501 949, necesarios para cubrir las operaciones durante tres meses.

- Se afirma que el proyecto es económica y financieramente viable, debido a que el VANE es de S/ 162084 y el VANF es de S/ 325 140, mayores a cero. Además, la tasa de retorno TIRE es de 28,30 \% y el TIRF es de 35,04\%, mayores que el costo de oportunidad de capital $(\mathrm{Cok}=25,01 \%)$. El ratio de beneficio-costo del proyecto es de 1,4 y presenta un periodo de recupero en el cuarto año.

- Con respecto del análisis de sensibilidad, ante los cambios de las tres variables propuestas en los tres escenarios posibles, se obtiene un proyecto rentable económica y financieramente.

\section{REFERENCIAS}

Encina Zelada, C., \& Carpio Rivadeneira, L. (2011). Máxima retención de ácido ascórbico, compuestos bioactivos y capacidad antioxidante en el néctar de Ingeniería Industrial, 225-245.

FAO. (2007). Guía de Campo de los Cultivos Andinos. Recuperado de http://www.fao.org/docrep/010/ai185s/ai185s.pdf

Gestión. (4 de abril de 2014). Consumo: Desaceleración, migración o cautela. Recuperado de http://gestion.pe/opinion/consumo-desaceleracion-migracion-cautela-2107621 
Gestión. (agosto, 2014). Mypes de nueve regiones participan en Expoalimentaria 2014. Recuperado de http://gestion.pe/economia/producemypes-nueve-regiones-participan-expoalimentaria-2014-2107073

Gestión. (diciembre, 2014). Terrenos en nueva zona industrial al este de Lima cuestan US\$120 por metro cuadrado. Recuperado de https://www.google.com.pe/search?q=Terrenos+en+nueva+zona+i ndustrial+al+este+de+Lima+cuestan+US\%24+120+por+metro+ cuadrado\&oq=Terrenos+en+nueva+zona+industrial+al+este+de + Lima+cuestan+US\%24+120+por+metro+cuadrado\&aqs=chrom e..69i57.88980j0j4\&sourceid=chrome

Instituto de Investigación Agropecuaria. (2015). Centro de Investigación Especializado en Agricultura del Desierto y Altiplano (CIE), "Potencia forestal Chile", Cultivo del tumbo en la precordillera de Putre. Recuperado de http://platina.inia.cl/ururi/informativos/ Informativo_INIA_Ururi_54.pdf

Instituto de Investigación Científica Agropecuaria. (s.f.). La curuba y su cultivo. Recuperado de http://sian.inia.gob.ve/repositorio/ revistas_tec/FonaiapDivulga/fd59/curuba.html

Ministerio de Economía y Finanzas. (2014). Marco macroeconómico multianual 2012-2014. Recuperado de https://www.mef.gob.pe/ contenidos/pol_econ/marco_macro/MMM2012_2014.pdf

Oswaldo Flores, A. (2002). Técnicas de propagación y mejoramiento del cultivo del tumbo (Passiflora Mollisima, L) en Tarata. (Trabajo de investigación). Recuperado del sitio de internet de la Universidad Nacional Jorge Basadre Grohmann: http://www.unjbg.edu.pe/ coin2/pdf/c\&d_9_art_29.pdf

Pardo Delgado, J., y Urquizo Baldárrago, K. (2014). Estudio de prefactibilidad para la implementación de una planta de elaboración de bebida de papaya (Carica papaya) con linaza (Linum usitatissimum). (Tesis para optar el título de Ingeniero Industrial). Universidad de Lima.

Salud y Seguridad en el Trabajo. (2013). Normas OHSAS 18001. Recuperado de http://www.bsigroup.com/es-ES/Seguridad-y-Saluden-el-Trabajo-OHSAS-18001/

Salud, Dinero \& Amor. (2008). Tumbo : Fruto de bajas calorías, evita cálculos renales, malestares urinarios y estomacales. Recuperado 
de https://plantitas.wordpress.com/2008/01/11/tumbo-fruto-de-babajas-caloras-evita-los-clculos-renales-malestares-urinarios-yestomacales/

Sule Dileep, R.(2001). Instalaciones de manufactura: Ubicación, planeación y diseño. México: Thomson Learning.

Universidad Nacional del Callao. (2013). Vitamina C en la Passiflora Mollisima (Tumbo andino). Recuperado de http://www.unac. edu.pe/documentos/organizacion/vri/cdcitra/Informes_Finales_ Investigacion/IF_OCTUBRE_2012/IF_LEON\%20ROMANI_ FIQ/INFORME\%20FINAL.pdf. 
